Journal of Animal and Veterinary Advances 11 (14): 2403-2408, 2012

ISSN: $1680-5593$

(C) Medwell Journals, 2012

\title{
Establishment and Evaluation of a New Method Tissue Re-Shift for Isolation of Goat Mammary Epithelial Cells
}

\author{
Xueqing Liu, Hao Yang, Jiayin Peng, Qiuju Chen and Binyun Cao \\ College of Animal Science and Technology, Northwest A\&F University, \\ 22 Xinong Road, 712100 Yangling, Shaanxi Province, People's Republic of China
}

\begin{abstract}
Isolation and culture of mammary epithelial cells in vitro is of great significance for further study of the mechanism of lactation, verification of mammary gland expression vector and preparation of mammary gland bioreactor. In this study, in order to obtain an efficient method to culture Goat Mammary Gland Epithelial (GMGE) cells with high purity in vitro, researchers successfully constructed a new method called Tissue Re-Shift Method. About 5 methods which include Tissue Explant Culture Method, Tissue Re-Shift Method, Trypsin Digestion Method, Collagenase Digestion Method and the Combination of Collagenase digestion with Tissue Adherence Method were applied and compared. In spite of no epithelial cells cultured by Trypsin Digestion Method and low capability of proliferation by combination of collagenase digestion with Tissue Adherence Method, a considerable number of GMGE cells were collected by another 3 methods. By the detection of immunofluorescence, Tissue Re-Shift Method can obtain pure GMGE cells in the shortest time. Biological characteristics of GMGE cells isolated from above 3 methods were evaluated by cell population doubling time and cell growth curves. The results indicated that cell growth curves of 3 methods showed typical S type and GMGE cells isolated from Tissue Re-Shift Method had maximum proliferative capacity. Finally, the existence of the $\beta$-casein verified that they still had the high ability of lactation in the primary culture. In conclusion, Tissue Re-Shift Method was an ideal way to obtain GMGE cells for further study.
\end{abstract}

Key words: Goat mammary gland epithelial cells, Tissue Re-Shift Method, immunofluorescence, growth curve, cell population doubling time, $\beta$-casein

\section{INTRODUCTION}

Proliferation and differentiation of mammary gland cells runs through the whole process of mammary gland development and lactation. Many predecessors have done a large number of researches on the proliferation and differentiation of mammary epithelial cell at the cellular level (Jehn et al., 1992; Desrivieres et al., 2003; Cucuzza et al., 2008). Primary mammary epithelial cells cultured in vitro conditions are similar to the physiological characteristics of breast tissue (Andersen and Larson, 1970; Larson, 1976; Haeuptle et al., 1983) which can overcome some problems difficult to study in vivo such as drug detection, virus infection, cellular characteristics and so on.

During passage, some characteristics of breast epithelial cells change but they still have the ability of lactation in primary culture (Haeuptle et al., 1983; Romanov et al., 2001). So, the isolation and culture in vitro of mammary epithelial cells is of great significance for further study of the mechanism of lactation, verification of mammary gland expression vector and preparation of mammary gland bioreactor. Whether primary culture of Mammary Gland Epithelial (MGE) cells is successful depends largely on the availability of a sufficient number of good vitality epithelial cells (Chavis and Anderson, 1988). Currently, there are many approaches to obtain epithelial cells from mammary gland such as tissue explant culture, enzymatic digestion, mechanical crushing and the separation from milk (Buehring, 1990; Pantschenko et al., 2000; Boutinaud et al., 2008; Hu et al., 2009). Separation from milk is demanding and difficult to control and inappropriate to the culture of Goat Mammary Gland Epithelial (GMGE) cells (Taylor-Papadimitriou et al., 1977; Ceriani et al., 1979; Gaffney, 1982). While methods tissue explant culture, enzymatic digestion and mechanical crushing always adulterate epithelial cells with fibroblast cells. At present, there has not been report about excellent culture system of GMGE cells yet. More research is urgently needed to screen out a quick method to obtain purified primary MGE cells and then establish a mature

Corresponding Author: Binyun Cao, College of Animal Science and Technology, Northwest A\&F University, 712100 Yangling, Shaanxi, People's Republic of China 
GMGE line by passaging. This study compared culture effect of five methods tissue explant culture, tissue re-shift, trypsin digestion, collagenase digestion and collagenase digestion with tissue explant culture to obtain GMGE cells. The purpose of this research is to establish a rapid and efficient GMGE culture system and provide a technical foundation for establishment of GMGE lines and preparation of mammary gland bioreactor.

\section{MATERIALS AND METHODS}

General reagents: The GMGE cells culture medium was composed of D-MEM/F12 (GIBCO), 10\% Fetal Bovine Serum (FBS, Hyclone), $1 \mu \mathrm{g} \mathrm{mL}^{-1}$ Hydrocortisone (Sigma), $5 \mu \mathrm{g} \mathrm{mL}^{-1}$ Insulin (Sigma), $10 \mathrm{ng} \mathrm{mL}^{-1}$ Insulin-like Growth Factor-1 (IGF-1, Sigma), $10 \mathrm{ng} \mathrm{mL}^{-1}$ Epithelial Growth Factor (EGF, Sigma), $100 \pi \mathrm{JL}^{-1}$ Penicillin (GIBCO) and $100 \mathrm{IU} \mathrm{mL}^{-1}$ Streptomycin (GIBCO). The Phosphate Buffered Saline (PBS) was composed of $8.00 \mathrm{~g} \mathrm{~L}^{-1} \mathrm{NaCl}$, $0.2 \mathrm{~g} \mathrm{~L}^{-1} \mathrm{Kcl}, 2.89 \mathrm{~g} \mathrm{~L}^{-1} \mathrm{Na}_{2} \mathrm{HPO}_{4} \cdot 12 \mathrm{H}_{2} \mathrm{O}, 0.2 \mathrm{~g} \mathrm{~L}^{-1}$ $\mathrm{KH}_{2} \mathrm{PO}_{4}, 100 \mathrm{IU} \mathrm{mL}^{-1}$ Penicillin and $100 \mathrm{IU} \mathrm{mL}^{-1}$ Streptomycin. The GMGE cells trypsin digestive medium was composed of PBS, $0.25 \%$ trypsin (GIBCO) and $0.02 \%$ Ethylene Diamine Tetraacetic Acid (EDTA). The GMGE cells collagenase digestive medium was composed of PBS and $0.2 \%$ collagenase (Sigma).

Total RNA was extracted from cells according to the instructions of RNAiso Plus (Takara, Dalian, China). First strand cDNA synthesis was preformed using OligodT according to the instructions of TIANScript cDNA kit (Tiangen, Beijing, China). Reagents used in immunofluorescence of the GMGE cell were Mouse Anti-Cytokeratin 18 (Anti-CK18, Boster, Wuhan, China), Cy3-sheep Anti-Mouse IgG kit (Boster, Wuhan, China) and Hoechst 33342 (Sigma).

Collection and preprocessing of goat mammary tissue: Goat mammary gland tissue was obtained from a 3 years old lactating Guanzhong diary goat after parturition which was placed under general anesthesia and terminated prior to waking according to a protocol approved by the Institutional Animal Care and Use Committee. The tissue was acquired in compliance with National Institutes of Health and Institutional Guidelines. Wash mammary tissue repeatedly with PBS and then mammary tissues were cut up into the size of $1 \mathrm{~mm}^{3}$ with ophthalmic scissors, finally wash the surface of the small tissue by PBS until no blood and milk.

In vitro culture of GMGE cells by 5 different methods: For tissue explant culture, the tissue blocks with the size of $1 \mathrm{~mm}^{3}$ were directly stuck to $60 \mathrm{~mm}$ culture dishes with a distance of $5 \mathrm{~mm}$ between each other so as to assure that there was enough space for the growth of the cells after they emigrated from the tissue explants and kept the even distribution of local cellular density. After the tissue explants were placed in the incubator of $37^{\circ} \mathrm{C}$, under $95 \%$ humidity and $5 \% \mathrm{CO}_{2}$ for $4 \mathrm{~h}$, the culture dishes were carefully transferred and added into $3 \mathrm{~mL}$ medium. The medium was changed every 2 days.

The rest tissue blocks were transferred to an erlenmeyer flask, mixed with supplemented enzyme digestion and incubated at $37^{\circ} \mathrm{C}$ in the incubator. The digestion time of collagense was $3.5 \mathrm{~h}$ and collection time of trypsin digestion was every $0.5 \mathrm{~h}$. Then, the explants were filtered through a $200 \mu \mathrm{m}$ mesh filter. The filtrate was transferred into a $10 \mathrm{~mL}$ centrifugation tube and centrifuged at $1000 \mathrm{r} \mathrm{min}^{-1}$ for $10 \mathrm{~min}$ and the supernatant was removed. After resuspended with $5 \mathrm{~mL}$ PBS, suspension was centrifuged at $1000 \mathrm{r} \mathrm{min}^{-1}$ for $10 \mathrm{~min}$. The supernatant was discarded and the pellet was resuspended with culture medium. Finally, cells were counted and seeding density of cells was $1.0 \times 10^{6}$ cells $\mathrm{mL}^{-1}$. The medium was changed every 2 days.

The tissue blocks digested by collagense were stuck to $60 \mathrm{~mm}$ culture dishes with a distance of $5 \mathrm{~mm}$ between each other and the next steps were carried out according to the description of tissue explant culture. The medium was changed every 2 days.

After 5 days culture, explants were surrounded by abundant adhering cells and inoculated into new $60 \mathrm{~mm}$ culture dishes. The tissue explants were placed in the incubator of $37^{\circ} \mathrm{C}$, under $95 \%$ humidity and $5 \% \mathrm{CO}_{2}$ for $2 \mathrm{~h}$. The culture dishes were carefully added into $3 \mathrm{~mL}$ medium and cultured continually. The medium was changed every 2 days.

Passage culture of GMGE cells: Primary cells were passaged into the $60 \mathrm{~mm}$ culture dishes when reached $70-80 \%$ adhere. Rinse the monolayer by gently rocking the flask back and forth with PBS. Aspirate PBS and pipette $0.25 \%$ trypsin $/ 0.02 \%$ EDTA onto the monolayer in the dishes. Incubate at $37^{\circ} \mathrm{C}$ for about $10 \mathrm{~min}$ until most cells were neither detached nor in clumps any more. Add the appropriate volume of cell suspension to two new dishes containing medium to ensure density at $1.0 \times 10^{4}$ cells $\mathrm{mL}^{-1}$. According to mammary epithelial cells and fibroblast cells by different trypsin sensitivity, two kinds of cells were isolated by phase contrast digestion obtaining to purified mammary epithelial cells and fibroblast cells.

Immunofluorescence of the GMGE cells: GMGE cells cultured by Tissue Explant Culture Method and 
Collagenase Digestion Method were purified owing to the different trypsin sensitivity from fibroblasts. GMGE cells derived form re-shift tissues were directly passaged into new dishes. Cells isolated and purified by these 3 methods were passaged into 6 well plates for the Immunofluorescence detection with Mouse Anti-CK18. Detailed procedure was preformed according to the instructions of Cy3-sheep Anti-Mouse IgG kit. Finally, cells nuclei were stained by Hoechst 33342 .

Detection of biological characteristics of GMGE cells: Cells were passaged when covered $80-90 \%$ of the total plate. Cells were digested by conventional digestion and counted. The cells were suspended in a base medium and seeded in 12 well plates and $35 \mathrm{~mm}$ dishes at a density of $5.0 \times 10^{4}$ cells $\mathrm{cm}^{-2}$, respectively for the calculation of population doubling time and drawing of growth curve. The doubling Time (T) of GMGE cells logarithmic growth phase was obtained using the following equation:

Where:

$$
\mathrm{T}=\mathrm{t}\left(\lg \mathrm{N}_{\mathrm{t}}-\lg \mathrm{N}_{0}\right) / \lg _{2}
$$

$N_{t}=$ The average number of cell retrieved in logarithmic growth phase

$\mathrm{N}_{0}=$ The seeding cell number

$\mathrm{t}=$ The culture time

The shorter the population doubling time is the stronger the cell proliferation ability is. For the drawing of growth curve, the seeded cells in $35 \mathrm{~mm}$ dishes were divided into 10 groups (each group 3 dishes) in an incubator at $37^{\circ} \mathrm{C}$ under $5 \% \mathrm{CO}_{2}$ and $95 \%$ humidity. Then, the calculation the average of cells within each group was carried out at 1-10 days after cell seeding. A standard growth curve was constructed with cell cunts with culture time as the $\mathrm{X}$-axis and cell number as $\mathrm{Y}$-axis.

RT-PCR analysis of $\boldsymbol{\beta}$-casein expression: Total RNA of GMGE cells was extracted by Trizol and then PCR amplified target fragment (211 bp) of $\beta$-casein gene after reverse transcription. Primers were designed according to Capra hircus beta-casein CDS sequence (GenBank: EF558564.1). Sense primer: 5'-AGCCTGAAATAATGGG AGTC-3'; Antisense primer: 5'-GGAGGAAACATGAC GGTTG-3'. The reaction procedures as: $95^{\circ} \mathrm{C} 4 \mathrm{~min}$; 30 cycles of $94^{\circ} \mathrm{C} 30 \mathrm{sec}, 55^{\circ} \mathrm{C} 30 \mathrm{sec}, 72^{\circ} \mathrm{C} 30 \mathrm{sec}$; $72^{\circ} \mathrm{C} \quad 10 \mathrm{~min}$. Amplification was analyzed by electrophoresis in $1 \%$ agarose.

\section{RESULTS AND DISCUSSION}

Morphology of cells cultured by 5 different methods: About 5 days after direct explantation, fibroblast cells developed from connective tissue in radial pattern. Total 8 days later, the epithelial cells grow outward along the tissue in polygon and connect tightly white each other into the film, the halo grows around the tissue form the growth membrane (Fig. 1a).

Inoculate the digested cells and observe them at the next day. Fibroblast cells were derived after digestion by trypsin for $2 \mathrm{~h}$ meanwhile no epithelial cells were found. There were a large number of non-adherent cells suspending in the culture medium and still no epithelial cells after cultured 3 days later. After digested by collagenase for $3.5 \mathrm{~h}$, epithelial cells were mainly derived. Epithelial cells were polygonal and there were a small
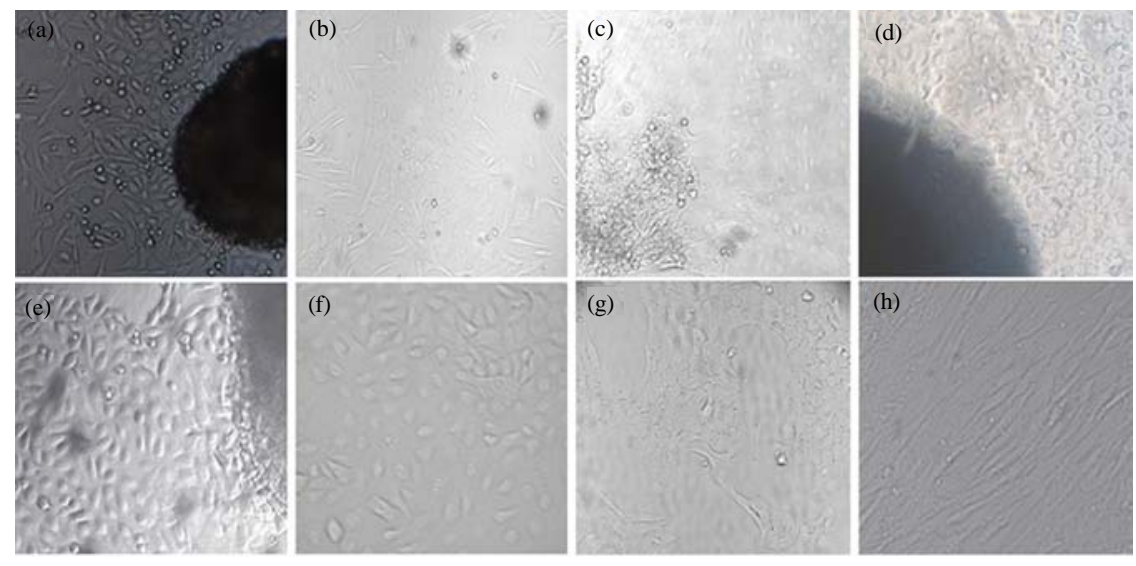

Fig. 1: Morphologiacal observation of the cultured GMGE and fibroblasts cells (200x); a) Tissue both grown fibroblast and epithelial cells; b) Digested by collagenase epithelial cells and fibroblasts mixed grown; c) Epithelial cells grown after flocculent tissue digestion adherent; d) Epithelial cells grown after combination of collagenase digestion with tissue adherence; e) Epithelial cells grown after re-shifted issue; f) Purified epithelial cells; g) Reticulation formed among epithelial cells; h) Purified fibroblasts 
number of fibroblasts on the edge of epithelial cells (Fig. 1b). The flocculent tissues which were digested off grew vigorous after adherent to the culture dishes (Fig. 1c).

When Combining Method Collagenase Digestion with Explant Culture, the epithelial cells grew around the flocculent tissues 3 days after the inoculation. However, there were a lot of vacuoles among the cells and the cells grew slowly and tended to die (Fig. 1d).

In re-shift treatment, 2 days after the explants cultured in new culture dishes, large numbers of epithelial cells densely distributed around the explants and the cells were pure and uniform in morphology and there were no fibroblasts (Fig. 1e). While 5 days later, the epithelial cells covered $80 \%$ of the dishes.

The cells obtained by all 5 methods were passaged by trypsin digestation to make the epithelial cells separate from fibroblasts. The epithelial cells grew in polygonal and connected with each other tightly into a film (Fig. 1f). It was a typical structure that epithelial cells bumped and formed the net structure (Fig. 1g). Fibroblasts grew fusiform in morphology (Fig. 1h). The passage cell obtained by collagenase digestion coupled with explant culture could not adherent to the culture dishes.

Immunofluorescence of the GMGE cells: Immunofluorescence results of the GMGE cells isolated by tissue explant culture, tissue re-shift and collagenase digestion showed that all the 3 methods could obtain a certain amount of GMGE cells but at least 5 times of passage were needed for cells isolated by Tissue Explant Culture Method and Collagenase Digestion Method. While cells isolated by tissue re-shift were almost GMGE cells (Fig. 2a-c). It is indicated that Tissue Re-Shift Method can obtain pure GMGE cells in the shortest time.

Detection of biological characteristics of GMGE cells: As shown in Table 1, the cell doubling time of GMGE cells isolated by Tissue re-shift method was the shortest so the proliferative capacity of GMGE cells derived from re-shift tissues was higher than another 2 methods.

As shown in Fig. 3, growth of primary cells isolated by tissue explant culture, tissue re-shift and collagenase digestion were in good condition and cell growth curves showed typical S type and were in line with the general laws of cell growth.

RT-PCR analysis of $\boldsymbol{\beta}$-casein expression: A fragment with the length of $211 \mathrm{bp}$ was successfully amplified from the cultured GMGE cells isolated by tissue explant culture, tissue re-shift and collagenase digestion (Fig. 4) which verified the existence of the $\beta$-casein. It is clear that they still had the ability of lactation in the primary culture.
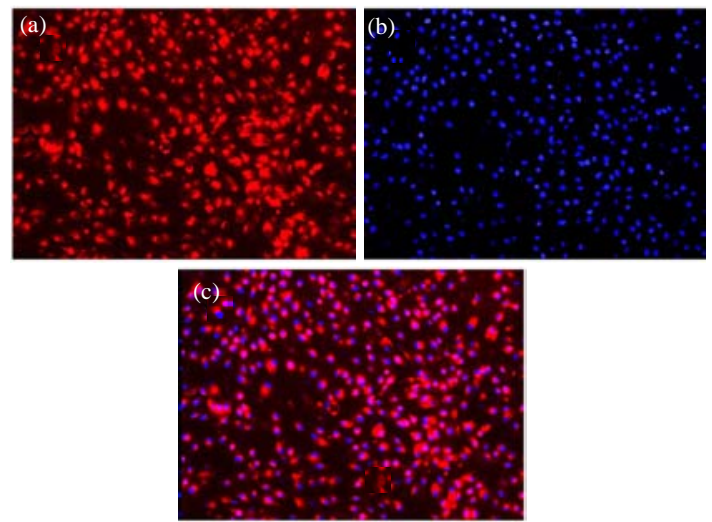

Fig. 2: Immunofluorescence of the GMGE cell momolayer (200x). After $24 \mathrm{~h}$ of passage; the mammary gland epithelial cell momolayer was stained with antibody CK18; a) Positive staining of CK18; b) Cells nuclei stained by Hoechst 33342; c) Merged images of $a$ and $b$ labeling

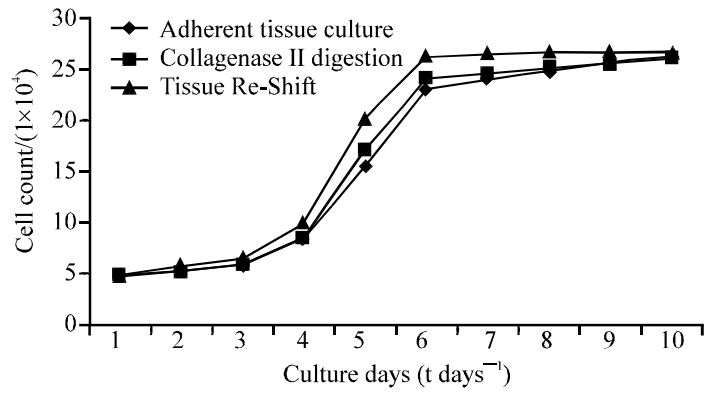

Fig. 3: GMGE cell growth curves of different culture methods; a) GMGE cell growth curves of Tissue Re-Shift Method; b) GMGE cell growth curves of adherent tissue culture; c) GMGE cell growth curves of collagenase digestion

Table 1: Different methods of cell culture doubling time

\begin{tabular}{lccc}
\hline Culture Methods & $\mathrm{N}_{\mathrm{t}}\left(\times 10^{4}\right)$ & $\mathrm{N}_{0}\left(\times 10^{4}\right)$ & $\mathrm{T}$ \\
\hline Adherent Tissue Culture & 30.12 & 5.000 & 31.30 \\
Collagenase Digestion & 31.25 & 5.000 & 30.23 \\
Tissue Re-Shift & 33.43 & 5.000 & 26.89 \\
\hline
\end{tabular}

Morphometric study of MGE cells had demonstrated that some cells showed a cobblestone-like and some were polygonal (Hamamoto et al., 1988). Researchers also found these two forms of cells in this study.

There are so many methods to isolate GMGE cells but usually Enzymatic Digestion and Tissue Explant Culture Method. In general, enzymes used for enzymatic digestion are as follows: trypsin, collagenase, hyaluronidase, protease enzyme and so on (Ahn et al., 1995; Sun et al., 2006; Freshney, 2010). Among them, the most commonly used were trypsin and collagenase. Living cells isolated by trypsin in this study were almost fibroblasts. 


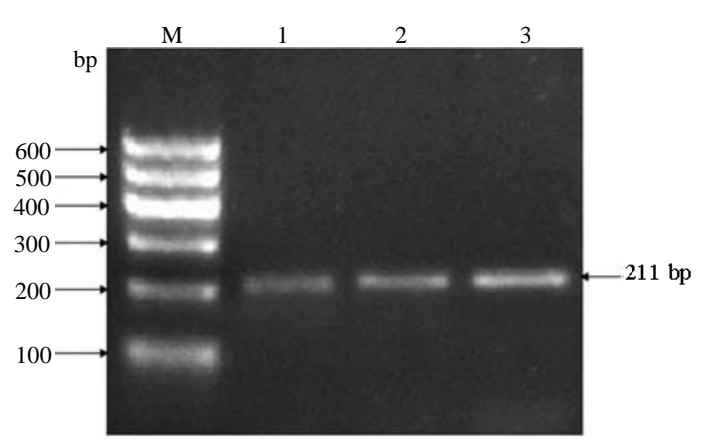

Fig. 4: RT-PCR analysis of $\beta$-casein gene. M: Marker; 1 : PCR product of $\beta$-casein gene in Adherent Tissue Culture Method; 2 : PCR product of $\beta$-casein gene in Collagenase Digestion Method; 3: PCR product of $\beta$-casein gene in Tissue Re-Shifts Method

A few of fibroblasts were also incorporated into epithelial cells when cells isolated by Collagenase Digestion Method. A lot of mammary epithelial cells in good shape and with proliferative ability were obtained by Tissue Explant Method but fibroblasts were firstly derived from the tissue in the 1st several days and this process generrally demanded a long period. Studies had shown that fibroblasts were more sensitive than epithelial cells (Berman et al., 1980) so, the fibroblasts primarily dropped off while epithelial cells remained in the sidewall by controlling the appropriate digestion time.

Extending digestion time purified epithelial cells were collected. But the purification process was time consuming and could not be achieved in a short time. Therefore, this study combined Collagenase Digestion with Tissue Explant Culture Method aiming to quickly obtain GMGE cells. But cells obtained by this method were incapable of proliferation. It suggested that collagenase might have damage to the physiological characteristics of cells. As shown in this research, researchers explanted the tissues when they were surrounded by abundant adhering cells after 5 days tissue explant culture.

Researchers defined this method as Tissue Re-Shift which was firstly proposed. By this new method, a considerable number of purified GMGE cells were isolated from goat mammary gland in a short time. GMGE cells cultured by Tissue Explant Culture Method and Collagenase Digestion Method were purified owing to the different trypsin sensitivity from fibroblasts.

GMGE cells derived form re-shift tissues were directly passaged into new dishes. Cells isolated and purified by these 3 methods were identified by immunofluorescence. It shown that at least 5 times of passage were needed for cells isolated by Tissue Explant Culture Method and
Collagenase Digestion Method and cells around tissues re-shifted were nearly GMGE cells. So, it confirmed the truth of superiority of Tissue Re-Shift Method.

By the calculation of population doubling time and the drawing of growth curve, it was apparent that all cells isolated and purified by 3 methods were high capable of proliferation and cells passaged from Tissue Re-Shift Method had a superior performance.

Finally, researchers determined the expression states of $\beta$-casein in cultured cells by RT-PCR (Riley et al., 2009; Monzani et al., 2011). $\beta$-casein still expressed at a high level. It demonstrated cells remained a strong capability of secretion.

Considering all results, researchers concluded that Tissue Re-Shift Method was the most effective approach to obtain a great amount GMGE cells for subsequent research.

\section{CONCLUSION}

In this research, research compared 5 methods isolating GMGE cells. The results showed that Tissue Re-Shift Method, research constructed was the fastest accessing to purified GMGE cells; cells isolated and purified by Tissue Re-Shift Method were highest capable of proliferation; cells remained a strong capability of secretion.

It kept GMGE cells closer to the physiological characteristics of breast tissue than other methods which could overcome some problems difficult to study in vivo such as drug detection, virus infection, cellular characteristics and so on. So, Tissue Re-Shift Method was an ideal way to obtain GMGE cells for further study of the mechanism of lactation, verification of mammary gland expression vector and preparation of mammary gland bioreactor.

\section{ACKNOWLEDGEMENTS}

This research was supported by grants from the The 12th 5 years Plan of National Science and Technology Project in Rural Areas (Grant No.: 2011BAD28B05-3) and Planning Projects on Integration and Innovation of Science and Technology in Shaanxi Province (Grant No.: 2011KTCL02-09).

\section{REFERENCES}

Ahn, J.Y., N. Aoki, T. Adachi, Y. Mizuno, R. Nakamura and T. Matsuda, 1995. Isolation and culture of bovine mammary epithelial cells and establishment of gene transfection conditions in the cell. Biosci. Biotechnol. Biochem., 59: 59-64. 
Andersen, C.R. and B.L. Larson, 1970. Comparative maintenance of function in dispersed cell and organ cultures of bovine mammary tissue. Exp. Cell Res., 61: 24-30.

Berman, J.J., C. Tong and G.M. Williams, 1980. Differences between rat liver epithelial cells and fibroblast cells in sensitivity to 8-Azaguanine. In vitro, 16: 661-668.

Boutinaud, M., M.H. ben Chedly, E. Delamaire and J. Guinard-Flament, 2008. Milking and feed restriction regulate transcripts of mammary epithelial cells purified from milk. J. Dairy Sci., 91: 988-998.

Buehring, G.C., 1990. Culture of mammary epithelial cells from bovine milk. J. Dairy Sci., 73: 956-963.

Ceriani, R.L., J. Taylor-Papadimitriou, J.A. Peterson and P. Brown, 1979. Characterization of cells cultured from early lactation milks. In vitro, 15: 356-362.

Chavis, D.D. and R.R. Anderson, 1988. Viability maintenance of mammary epithelial cells in vitro. Cell Biol. Int. Rep., 12: 155-165.

Cucuzza, L.S., M. Motta, S. Miretti, P. Accornero and M. Baratta, 2008. Curcuminoid-phospholipid complex induces apoptosis in mammary epithelial cells by STAT-3 signaling. Exp. Mol. Med., 40: 647-657.

Desrivieres, S., T. Prinz, N.C.P. Laria, M. Meyer and G. Boehm et al., 2003. Comparative proteomic analysis of proliferating and functionally differentiated mammary epithelial cells. Mol. Cell Proteomics., 2: 1039-1054.

Freshney, R.I., 2010. Culture of Animal Cells: A Manual of Basic Technique and Specialized Applications. 6th Edn., Wiley-Blackwell, New Jersey.

Gaffney, E.V., 1982. A cell line (HBL-100) established from human breast milk. Cell Tissue Res., 227: 563-568.

Haeuptle, M.T., Y.L. Suard, E. Bogenmann, H. Reggio, L. Racine and J.P. Kraehenbuhl, 1983. Effect of cell shape change on the function and differentiation of rabbit mammary cells in culture. J. Cell Biol., 96: 1425-1434.

Hamamoto, S., W. Imagawa, J. Yang and S. Nandi, 1988. Morphogenesis of mouse mammary epithelial celling growing within collagen gels: Ultrastuctural and immunocytochemical characterization. Cell Differ., 22: 191-201.
Hu, H., J. Wang, D. Bu, H. Wei, L. Zhou, F. Li and J.J. Loor, 2009. In vitro culture and characterization of a mammary epithelial cell line from Chinese Holstein dairy cow. PLoS One, 4: e7636-e7636.

Jehn, B., E. Costello, A. Marti, N. Keon and R. Deane et al., 1992. Overexpression of Mos, Ras, Src and Fos inhibits mouse mammary epithelial cell differentiation. Cell. Mol. Biol., 12: 3890-3902.

Larson, B.L., 1976. Comparative production of beta lactoglobulin and orotic acid with lactose in bovine mammary cell cultures: Effects of cell density and constituent inhibition. J. Dairy Sci., 59: 1881-1889.

Monzani, P.S., F.F. Bressan, L.G. Mesquita, J.R. Sangalli and F.V. Meirelles, 2011. $\beta$-casein gene expression by in vitro cultured bovine mammary epithelial cells derived from developing mammary glands. Genet. Mol. Res., 10: 604-614.

Pantschenko, A.G., M.R. Barber, J. Woodcock-Mitchell, S.L. Bushmich and T.J. Yang, 2000. Establishment and characterization of a Caprine Mammary Mammary Epithelial Cell line (CMEC). In vitro Cell. Dev. Biol. Anim., 36: 351-356.

Riley, L.G., M. Gardiner-Garden, P.C. Thomson, P.C. Wynn, P. Williamson, H.W. Raadsma and P.A. Sheehy, 2009. The influence of extracellular matrix and prolactin on global gene expression profiles of primary bovine mammary epithelial cells in vitro. Anim. Genet., 41: 55-63.

Romanov, S.R., B.K. Kozakiewicz, C.R. Holst, M.R. Stampfer, L.M. Haupt and T.D. Tlsty, 2001. Normal human mammary epithelial cells spontaneously escape senescence and acquire genomic changes. Nature, 409: 633-637.

Sun, Y.L., C.S. Lin and Y.C. Chou, 2006. Establishment and characterization of a spontaneously immortalized porcine mammary epithelial cell line. Cell Biol. Int., 30: $970-976$.

Taylor-Papadimitriou, J., M. Shearer and R. Tilly, 1977. Some properties of cells cultured from early-lactation human milk. J. Natl. Cancer Inst., 58: 1563-1571. 